Article

\title{
Condensation of 2-naphtol with arylaldehydes using acetic acid functionalized ionic liquids as highly efficient and reusable catalysts
}

\author{
Ahmad Reza Moosavi-Zare a,*, Mohammad Ali Zolfigol b,\#, Omid Khaledian b, Vahid Khakyzadeh ${ }^{\mathrm{b}}$ \\ a Department of Chemistry, University of Sayyed Jamaleddin Asadabadi, Asadabad, 6541835583, Iran \\ b Faculty of Chemistry, Bu Ali Sina University, Hamedan, 6517838683, Iran
}

A R T I C L E I N F O

Article history:

Received 3 December 2013

Accepted 13 January 2014

Published 20 April 2014

\section{Keywords:}

14-Aryl-14H-dibenzo[a,j]xanthene

Acetic acid functionalized ionic liquid

2-Naphthol

Aldehyde

Catalyst

Solvent-free

\section{A B S T R A C T}

An efficient solvent-free protocol for the synthesis of 14-aryl-14H-dibenzo[a,j]xanthenes from the condensation of 2-naphthol with arylaldehydes, using acetic acid functionalized imidazolium salts (1-carboxymethyl-3-methylimidazolium bromide ([cmmim]Br) and 1-carboxymethy1-3-methylimidazolium tetrafluoroborate $\left([\mathrm{cmmim}] \mathrm{BF}_{4}\right)$ as reusable catalysts, has been developed. The turn over frequency on the catalysts is several times higher than the other previously reported catalysts. Also, thermal gravimetric analysis and powder X-ray diffraction pattern of the catalysts have been studied.

(C) 2014, Dalian Institute of Chemical Physics, Chinese Academy of Sciences. Published by Elsevier B.V. All rights reserved.

\section{Introduction}

Ionic liquids (based imidazolium or other organic cations) have received considerable interest as eco-friendly solvents, catalysts, and reagents in organic synthesis because of their unique properties, such as low volatility, non-flammability, high thermal stability, negligible vapor pressure, and the ability to dissolve a wide range of materials [1-9]. Among them, Brønsted acidic ionic liquids, which add the useful characteristics of solid acids and mineral liquid acids, have been designed to replace traditional mineral acids like $\mathrm{H}_{2} \mathrm{SO}_{4}$ and $\mathrm{HCl}$ in chemical procedures [10-25].

The 14-aryl-14H-dibenzo[a,j]xanthene group is a key structural element of many biologically active compounds, such as antibacterials [26], antivirals [27], anti-inflammatory agents [28], and in photodynamic therapy [29]. Xanthene-based com- pounds have also been investigated for agricultural bactericide activity and some other benzoxanthenes have found applications in industry as dyes for use in lasers [30] and as fluorescent materials for visualization of biomolecules [31]. Xanthene dyes can be extracted from soil and plants, such as Indigofera Longeracemosa [32,33]. Some methods for the synthesis of 14-aryl-14H-dibenzo[a,j]xanthenes have been reported by condensing 2-naphthol with aldehydes in the presence of a protic or Lewis acid catalyst [34-45]. However, these catalytic systems suffer from some limitations, such as long reaction time, high catalyst loadings, the use of toxic solvents, or special apparatus. The search for milder and more environmentally benign conditions would therefore be of great benefit in the synthesis of these compounds.

Here, the acetic acid functionalized imidazolium salts 1-carboxymethyl-3-methylimidazolium bromide ([cmmim]Br)

\footnotetext{
* Corresponding author. Tel: +98 8123237450; Fax: +98 8123237451; E-mail: moosavizare@yahoo.com

\# Corresponding author. Tel: +98 8118282807; Fax: +98 8118257407; E-mail: mzolfigol@yahoo.com

This work was supported by Bu-Ali Sina University and University of Sayyed Jamaleddin Asadabadi, Asadabad.

DOI: 10.1016/S1872-2067(14)60030-9 | http://www.sciencedirect.com/science/journal/18722067 | Chin. J. Catal., Vol. 35, No. 4, April 2014
} 
and 1-carboxymethy1-3-methylimidazolium tetrafluoroborate ([cmmim] $\left.\left[\mathrm{BF}_{4}\right]\right)$ as highly efficient and reusable catalysts for the preparation of 14-aryl-14H-dibenzo[a,j]xanthene derivatives was reported. Their effectiveness has been tested in the reaction of 2-naphthol with aromatic aldehydes under solvent-free conditions (Scheme 1).

\section{Experimental}

\subsection{General}

All chemicals were purchased from Merck or Fluka Chemical Companies. The products were identified by comparison of their melting points (MP) and spectral data in the literature. Acetic acid functionalized ionic liquids were prepared according to literature procedures $[44,45]$. The progress of each catalyzed reaction was monitored by TLC using silica gel SIL G/UV 254 plates. The ${ }^{1} \mathrm{H}$ NMR (400 or $300 \mathrm{MHz}$ ) and ${ }^{13} \mathrm{C}$ NMR (100 or $75 \mathrm{MHz}$ ) were run on a Bruker Avance DPX-250 FT-NMR spectrometer ( $\delta$ in ppm). Melting points were recorded on a Büchi B-545 apparatus in open capillary tubes.

\subsection{General procedure for the synthesis of 14-aryl-14H-dibenzo[a,j]xanthenes}

A mixture of arylaldehyde (1 mmol), 2-naphtol (2 mmol) and catalyst $\left([\mathrm{cmmim}] \mathrm{Br}\right.$ or $\left[\mathrm{cmmim}^{\mathrm{c}} \mathrm{BF}_{4}\right)(0.1 \mathrm{mmol}, 10$ mol\%) was stirred at $115{ }^{\circ} \mathrm{C}$. When the reaction was complete, as judged by TLC, $\mathrm{H}_{2} \mathrm{O}(1 \mathrm{~mL})$ was added to the reaction mixture, stirred and refluxed for $3 \mathrm{~min}$. Then, the reaction mixture was filtered and all water in the filtrate was removed under reduced pressure to separate the catalyst from the crude product. The solid residue (crude product) was collected by filtration and recrystallized from hot ethanol (95\%) to give the pure product. The recovered catalyst was washed with $\mathrm{CHCl}_{3}$, dried under reduced pressure and reused for the next run.

14-phenyl-14H-dibenzo[a,j]xanthene (1b). IR (KBr, cm-1): 3075, 1621, 1592, 1513, 1243, 1152, 803, 765; ${ }^{1} \mathrm{H}$ NMR (DMSO$\left.\mathrm{d}_{6}, 400 \mathrm{MHz}\right): \delta 6.74(\mathrm{~s}, 1 \mathrm{H}), 6.97(\mathrm{t}, J=7.6 \mathrm{~Hz}, 1 \mathrm{H}), 7.14(\mathrm{t}, J=$ $7.6 \mathrm{~Hz}, 2 \mathrm{H}), 7.45(\mathrm{t}, J=7.2 \mathrm{~Hz}, 2 \mathrm{H}), 7.57(\mathrm{~d}, J=8.8 \mathrm{~Hz}, 2 \mathrm{H})$, 7.62-7.66 (m, 4H), 7.91-7.93 (m, 4H), 8.70 (d, J = $8.8 \mathrm{~Hz}, 2 \mathrm{H})$; ${ }^{13} \mathrm{C}$ NMR (DMSO-d6, $75 \mathrm{MHz}$ ): $\delta 37.0,117.9,118.1,123.8,124.9$, $126.7,127.4,128.4,128.8,129.0,129.4,131.1,131.3,146.0$, 148.4 .

14-(2-chlorophenyl)-14H-dibenzo[a,j]xanthene (2b). IR
(KBr, cm-1): 3057, 1622, 1592, 1515, 1247, 1141, 809, 745; ${ }^{1} \mathrm{H}$ NMR (DMSO-d, $300 \mathrm{MHz}): \delta 6.64(\mathrm{~s}, 1 \mathrm{H}), 6.91-7.03(\mathrm{~m}, 2 \mathrm{H})$, 7.27 (d, $J=7.7 \mathrm{~Hz}, 2 \mathrm{H}), 7.38-7.50(\mathrm{~m}, 5 \mathrm{H}), 7.57-7.70(\mathrm{~m}, 2 \mathrm{H})$, 7.76-7.90 (m, 4H), $8.54(\mathrm{~d}, J=8.4 \mathrm{~Hz}, 1 \mathrm{H}) ;{ }^{13} \mathrm{C}$ NMR (DMSO-d 6 , $75 \mathrm{MHz}$ ): $\delta 34.8,116.9,118.2,123.3,124.9,127.4,128.5,128.8$, 129.2, 129.8, 130.2, 130.3, 130.9, 131.4, 132.0, 143.2, 148.7.

14-(3-chlorophenyl)-14H-dibenzo[a,j]xanthene (3b). IR (KBr, $\mathrm{cm}^{-1}$ ): 3069, 1623, 1591, 1246, 1141, 813, 746; ${ }^{1} \mathrm{H}$ NMR (DMSO-d6, $300 \mathrm{MHz}): \delta 6.74(\mathrm{~s}, 1 \mathrm{H}), 7.01(\mathrm{~d}, J=8.1 \mathrm{~Hz}, 1 \mathrm{H})$, $7.13(\mathrm{t}, J=7.8 \mathrm{~Hz}, 1 \mathrm{H}), 7.42(\mathrm{t}, J=7.2 \mathrm{~Hz}, 2 \mathrm{H}), 7.52-7.66(\mathrm{~m}$, 6H), $7.91(\mathrm{~d}, J=8.7 \mathrm{~Hz}, 4 \mathrm{H}), 8.67(\mathrm{~d}, J=8.7 \mathrm{~Hz}, 2 \mathrm{H}) ;{ }^{13} \mathrm{C} \mathrm{NMR}$ (DMSO-d6, 75 MHz): $\delta$ 36.5, 117.2, 118.1, 123.7, 125.1, 126.8, 127.0, 127.5, 127.9, 129.1, 129.7, 130.7, 131.1, 131.2, 133.5, 148.2, 148.5 .

14-(4-chlorophenyl)-14H-dibenzo[a,j]xanthene (4b). IR (KBr, cm-1): 3076, 1622, 1593, 1515, 1254, 1153, 804, 745; ${ }^{1} \mathrm{H}$ NMR (DMSO-d6, $300 \mathrm{MHz}): \delta 6.76(\mathrm{~s}, 1 \mathrm{H}), 7.18(\mathrm{~d}, J=6.8 \mathrm{~Hz}$, 2H), 7.46-7.64 (m, 10H), 8.92 (d, $J=7.8 \mathrm{~Hz}, 2 \mathrm{H}), 8.66$ (d, $J=7.6$ $\mathrm{Hz}, 2 \mathrm{H}$ ); ${ }^{13} \mathrm{C}$ NMR (DMSO-d6, $75 \mathrm{MHz}$ ): $\delta 36.3,117.4,118.1$, 123.4, 123.7, 125.0, 127.4, 128.8, 129.1, 129.6, 130.1, 131.1, 131.2, 144.8, 148.4 .

14-(2,3-dichlorophenyl)-14H-dibenzo[a,j]xanthene (5b). IR (KBr, cm${ }^{-1}$ ): 3058, 1633, 1594, 1255, 1141, 965, 817, 748, 676; 1H NMR ( $\left.\mathrm{CDCl}_{3}, 300 \mathrm{MHz}\right): \delta 6.86(\mathrm{~s}, 1 \mathrm{H}), 7.11(\mathrm{t}, J=1.1 \mathrm{~Hz}, 1 \mathrm{H})$, $7.28(\mathrm{~s}, 1 \mathrm{H}), 7.34(\mathrm{~d}, J=8.0 \mathrm{~Hz}, 1 \mathrm{H}), 7.44-7.52(\mathrm{~m}, 4 \mathrm{H}), 7.65(\mathrm{t}, J$ $=7.9 \mathrm{~Hz}, 2 \mathrm{H}), 7.84(\mathrm{t}, J=8.6 \mathrm{~Hz}, 4 \mathrm{H}), 8.69(\mathrm{~d}, J=8.5 \mathrm{~Hz}, 2 \mathrm{H}) ;{ }^{13} \mathrm{C}$ NMR $\left(\mathrm{CDCl}_{3}, 300 \mathrm{MHz}\right): \delta 35.6,117.7,118.1,123.3,124.5$, 127.1, 128.1, 128.7, 129.2, 129.9, 130.8, 131.6, 146.4, 148.9, $155,7$.

14-(3-bromophenyl)-14H-dibenzo[a,j]xanthene (6b). IR (KBr, $\left.\mathrm{cm}^{-1}\right): 3065,1622,1591,1398,1239,1140,810,746 ;{ }^{1} \mathrm{H}$ NMR (DMSO-d, $300 \mathrm{MHz}): \delta 6.63(\mathrm{~s}, 1 \mathrm{H}), 6.90-7.02(\mathrm{~m}, 2 \mathrm{H})$, 7.26 (d, $J=7.7 \mathrm{~Hz}, 2 \mathrm{H}), 7.37-7.49(\mathrm{~m}, 5 \mathrm{H}), 7.58(\mathrm{t}, J=7.9 \mathrm{~Hz}$, 2H), 7.85-7.89 (m, 4H), $8.53(\mathrm{~d}, J=8.5 \mathrm{~Hz}, 1 \mathrm{H}) ;{ }^{13} \mathrm{C} \mathrm{NMR}$ (DMSO-d 675 MHz): $\delta 34.8,116.9,118.2,123.3,124.9,127.4$, 128.5, 128.8, 129.1, 129.8, 130.2, 130.3, 130.9, 131.4, 132.0, 143.2, 148.6 .

14-(4-bromophenyl)-14H-dibenzo[a,j]xanthene (7b). IR (KBr, cm-1): 3070, 1634, 1591, 1238, 1158, 940, 832, 740, 677; ${ }_{1} \mathrm{H} \mathrm{NMR}\left(\mathrm{CDCl}_{3}, 300 \mathrm{MHz}\right): \delta 6.46(\mathrm{~s}, 1 \mathrm{H}), 7.28-7.83(\mathrm{~m}, 10 \mathrm{H})$, 8.31 (s, 2H); ${ }^{13} \mathrm{C} \mathrm{NMR}\left(\mathrm{CDCl}_{3}, 300 \mathrm{MHz}\right): \delta 37.4,116.6,118.0$, $122.4,124.4,126.9,128.9,129.1,129.9,131.2,131.6,144.0$, 148.6.

14-(4-fluorophenyl)-14H-dibenzo[a,j]xanthene (8b). IR $\left(\mathrm{KBr}, \mathrm{cm}^{-1}\right): 3034,1632,1592,1502,1239,1095,813,743 ;{ }^{1} \mathrm{H}$

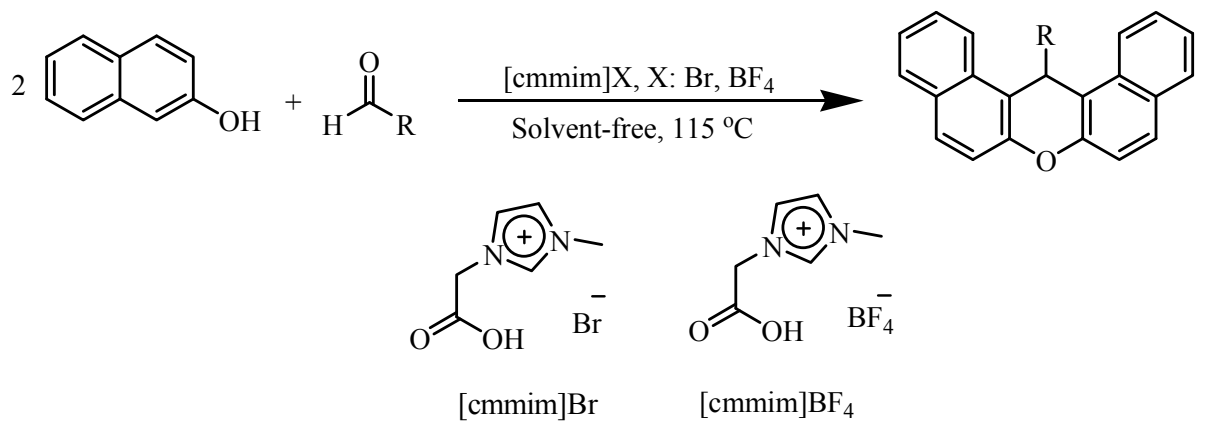

Scheme 1. Synthesis of 14-aryl-14H-dibenzo[a,j]xanthenes catalyzed by acetic acid functionalized imidazolium salts. 
NMR $\left(\mathrm{CDCl}_{3}, 300 \mathrm{MHz}\right): \delta 6.49(\mathrm{~s}, 1 \mathrm{H}), 6.83-6.89(\mathrm{~m}, 2 \mathrm{H})$, 7.43-7.53 (m, 6H), 7.59-7.65 (m, 2H), 7.81-7.88 (m, 4H), 8.36 (d, $J=8.5 \mathrm{~Hz}, 2 \mathrm{H}) ;{ }^{13} \mathrm{C} \mathrm{NMR}\left(\mathrm{CDCl}_{3}, 300 \mathrm{MHz}\right): \delta 37.2,115.2$, $115.5,117.1,118.0,122.5,124.3,126.9,128.9,129.0,129.6$, $129.7,131.0,131.3,140.8,148.7,159.5,162.8$.

14-(3-nitrophenyl)-14H-dibenzo[a,j]xanthene (9b). IR (KBr, $\mathrm{cm}^{-1}$ ): 3081, 1623, 1593, 1530, 1255, 1141, 808, 745; ${ }^{1} \mathrm{H}$ NMR (DMSO-d $6,300 \mathrm{MHz}): \delta 6.91(\mathrm{~s}, 1 \mathrm{H}), 7.11-7.25(\mathrm{~m}, 1 \mathrm{H})$, 7.43-7.48 (m, 2H), 7.55-7.71 (m, 4H), 7.88-8.03 (m, 7H), 8.66 (d, $J=8.4 \mathrm{~Hz}, 2 \mathrm{H}$ ); ${ }^{13} \mathrm{C}$ NMR (DMSO-d $6,75 \mathrm{MHz}$ ): $\delta 36.7,116.6$, 118.2, 123.6, 124.2, 125.2, 127.6, 129.1, 129.5, 130.0, 131.10, $131.19,146.3,148.4,153.1$.

14-(4-nitrophenyl)-14H-dibenzo[a,j]xanthene (10b). IR (KBr, cm-1): 3071, 1622, 1591, 1515, 1239, 1159, 827, 742; ${ }^{1} \mathrm{H}$ NMR (DMSO-d6, $300 \mathrm{MHz}): \delta 6.95(\mathrm{~s}, 1 \mathrm{H}), 7.43(\mathrm{t}, J=7.2 \mathrm{~Hz}$, $3 \mathrm{H}), 7.58-7.65(\mathrm{~m}, 4 \mathrm{H}), 7.81(\mathrm{~d}, J=7.5 \mathrm{~Hz}, 1 \mathrm{H}), 7.90-7.95(\mathrm{~m}$, $4 \mathrm{H}), 8.14(\mathrm{~d}, J=7.8 \mathrm{~Hz}, 1 \mathrm{H}), 8.45(\mathrm{~s}, 1 \mathrm{H}), 8.72(\mathrm{~d}, J=8.4 \mathrm{~Hz}, 2 \mathrm{H})$; ${ }^{13} \mathrm{C}$ NMR (DMSO-d6, $75 \mathrm{MHz}$ ): $\delta 36.3,116.9,118.2,122.0,122.5$, 123.6, 125.2, 127.7, 129.1, 130.0, 130.4, 131.11, 131.14, 134.7, $147.9,148.3,148.6$.

14-(4-Methylphenyl)-14H-dibenzo[a.j]xanthene (11b). IR (KBr, cm${ }^{-1}$ ): 3074, 1622, 1592, 1512, 1248, 1141, 812, 742; ${ }^{1} \mathrm{H}$ NMR (DMSO-d, $300 \mathrm{MHz}$ ): $\delta 2.01(\mathrm{~s}, 3 \mathrm{H}), 6.63(\mathrm{~s}, 1 \mathrm{H}), 6.90(\mathrm{~d}, J$ $=7.2 \mathrm{~Hz}, 2 \mathrm{H}), 7.40-7.61(\mathrm{~m}, 8 \mathrm{H}), 7.88(\mathrm{t}, J=2.7 \mathrm{~Hz}, 4 \mathrm{H}), 8.61$ (d, $J=8.7 \mathrm{~Hz}, 2 \mathrm{H}$ ); ${ }^{13} \mathrm{C}$ NMR (DMSO-d $6,75 \mathrm{MHz}$ ): $\delta 20.8,37.4$, $117.9,118.1,123.8,124.9,127.3,128.2,129.0,129.3,129.3$, 131.1, 131.3, 135.8, 143.0, 148.3 .

14-(4-hydroxy-3-methoxyphenyl)-14H-dibenzo[a,j]xanthen e (12b). IR ( $\left.\mathrm{KBr}, \mathrm{cm}^{-1}\right): 3475,3063,1592,1511,1242,1128$, 961, 817, 785, 694; ${ }^{1} \mathrm{H}$ NMR ( $\left.\mathrm{CDCl}_{3}, 300 \mathrm{MHz}\right): \delta 3.66(\mathrm{~s}, 3 \mathrm{H})$, $5.49(\mathrm{~s}, 1 \mathrm{H}), 6.45(\mathrm{~s}, 1 \mathrm{H}), 6.76(\mathrm{~d}, J=8.1 \mathrm{~Hz}, 1 \mathrm{H}), 6.87(\mathrm{~s}, 1 \mathrm{H})$, $7.19(\mathrm{~d}, J=8.1 \mathrm{~Hz}, 1 \mathrm{H}), 7.42-7.52(\mathrm{~m}, 4 \mathrm{H}), 7.61(\mathrm{t}, J=7.5 \mathrm{~Hz}$, 2H), $7.86(\mathrm{~m}, 4 \mathrm{H}), 8.42$ (d, $J=8.4 \mathrm{~Hz}, 2 \mathrm{H}) ;{ }^{13} \mathrm{C} \mathrm{NMR}\left(\mathrm{CDCl}_{3}, 300\right.$ $\mathrm{MHz}$ ): $\delta$ 37.6, 55,67, 110.6, 113.7, 117.5, 117.9, 120.9, 122.7, 124.3, 126.8, 128.8, 131.1, 131.4, 137.1, 144.1, 146.7, 148.7.

14-(4-benzophenyl)-14H-dibenzo[a,j]xanthene (13b). IR $\left(\mathrm{KBr}, \mathrm{cm}^{-1}\right): 3028,1620,1591,1514,1401,1242,1142,965$, 833, 740, 695; ${ }^{1} \mathrm{H}$ NMR $\left(\mathrm{CDCl}_{3}, 300 \mathrm{MHz}\right): \delta 6.56(\mathrm{~s}, 1 \mathrm{H})$, $7.29-7.66(\mathrm{~m}, 15 \mathrm{H}), 7.85(\mathrm{t}, J=9.7 \mathrm{~Hz}, 4 \mathrm{H}), 8.46(\mathrm{~d}, J=8.4 \mathrm{~Hz}$, $2 \mathrm{H}) ;{ }^{13} \mathrm{C} \mathrm{NMR}\left(\mathrm{CDCl}_{3}, 300 \mathrm{MHz}\right): \delta 37.7,117.2,118.0,122.7$, $124.3,126.8,126.9,127.0,127.2,128.6,128.8,131.1,131.4$, 139.2, 140.7, 144.0, 148.8 .

14-(phenethyl)-14H-dibenzo[a,j]xanthene (14b). IR (KBr, $\mathrm{cm}^{-1}$ ): 3055, 3022, 1622, 1592, 1516, 1488, 1459, 1158, 957; ${ }^{1} \mathrm{H}$ NMR $\left(\mathrm{CDCl}_{3}, 300 \mathrm{MHz}\right): \delta 2.39-2.51(\mathrm{~m}, 4 \mathrm{H}), 5.73(\mathrm{t}, J=4.0$ $\mathrm{Hz}, 1 \mathrm{H}), 6.87(\mathrm{~d}, J=6.6 \mathrm{~Hz}, 2 \mathrm{H}), 7.07-7.16(\mathrm{~m}, 3 \mathrm{H}), 7.48-7.57$ $(\mathrm{m}, 4 \mathrm{H}), 7.70(\mathrm{t}, J=7.8 \mathrm{~Hz}, 2 \mathrm{H}), 7.87(\mathrm{~d}, J=8.9 \mathrm{~Hz}, 2 \mathrm{H}), 7.97(\mathrm{~d}, J$ $=8.0 \mathrm{~Hz}, 2 \mathrm{H}), 8.34(\mathrm{~d}, J=8.5 \mathrm{~Hz}, 2 \mathrm{H}) ;{ }^{13} \mathrm{C} \mathrm{NMR}\left(\mathrm{CDCl}_{3}, 75 \mathrm{MHz}\right)$ : $\delta 31.1,37.3,116.1,117.7,122.5,124.3,125.6,126.8,128.1$, $128.2,128.5,129.0,131.2,131.4,141.9,150.1$.

14-(4-chloro-3-nitrophenyl)-14H-dibenzo[a,j]xanthene (15b). IR (KBr, cm-1): 3050, 1634, 1594, 1532, 1244, 1142, 802, 744; ${ }^{1} \mathrm{H} \mathrm{NMR}\left(\mathrm{CDCl}_{3}, 300 \mathrm{MHz}\right): \delta 6.56(\mathrm{~s}, 1 \mathrm{H}), 7.28(\mathrm{~d}, J=2.3$ $\mathrm{Hz}, 1 \mathrm{H}), 7.45-7.52(\mathrm{~m}, 4 \mathrm{H}), 7.62-7.67(\mathrm{~m}, 3 \mathrm{H}), 7.86$ (t, $J=8.4$ $\mathrm{Hz}, 4 \mathrm{H}), 8.05$ (d, $J=1.7 \mathrm{~Hz}, 1 \mathrm{H}), 8.25$ (d, $J=8.5 \mathrm{~Hz}, 2 \mathrm{H}) ;{ }^{13} \mathrm{C} \mathrm{NMR}$ $\left(\mathrm{CDCl}_{3}, 300 \mathrm{MHz}\right): \delta 37.1,115.4,118.1,121.8,124.7,124.9$, 125.2, 127.4, 129.1, 129.7, 130.9, 131.1, 132.0, 132.8, 145.2,
147.5, 148.8.

\section{Results and discussion}

Thermal gravimetric analysis (TGA) of the acetic acid functionalized imidazolium salts was also conducted from 25 to 600 ${ }^{\circ} \mathrm{C}$ at a rate of $10{ }^{\circ} \mathrm{C} / \mathrm{min}$ in $\mathrm{N}_{2}$ atmosphere (Fig. 1). As the TGA and differential thermal gravimetric (DTG) diagrams indicate, [cmmim] Br and [cmmim] $\mathrm{BF}_{4}$ decomposed in a single process at 230 and $250{ }^{\circ} \mathrm{C}$, respectively. Their TGA curves are similar to a single stage decomposition in which no intermediate is identified.

Powder X-ray diffraction (XRD) patterns of [cmmim]Br and [cmmim] $\mathrm{BF}_{4}$ were measured at $2 \theta=10^{\circ}-90^{\circ}$ (Fig. 2). As shown in Fig. 2, [cmmim] Br showed high crystallinity by the sharp, intense peaks at $2 \theta \approx 13.0^{\circ}, 15.7^{\circ}, 17.7^{\circ}, 20.8^{\circ}, 22.1^{\circ}, 23.3^{\circ}$, $26.2^{\circ}, 27.8^{\circ}, 29.5^{\circ}, 30.9^{\circ}, 40.9^{\circ}$, and several small lines from $50^{\circ}$ to $60^{\circ}$.

For $\left[\mathrm{cmmim} \mathrm{BF}_{4}\right.$, high crystallinity was also observed through the sharp peaks at $2 \theta \approx 12.8^{\circ}, 15.6^{\circ}, 17.3^{\circ}, 20.7^{\circ}, 21.9^{\circ}$,
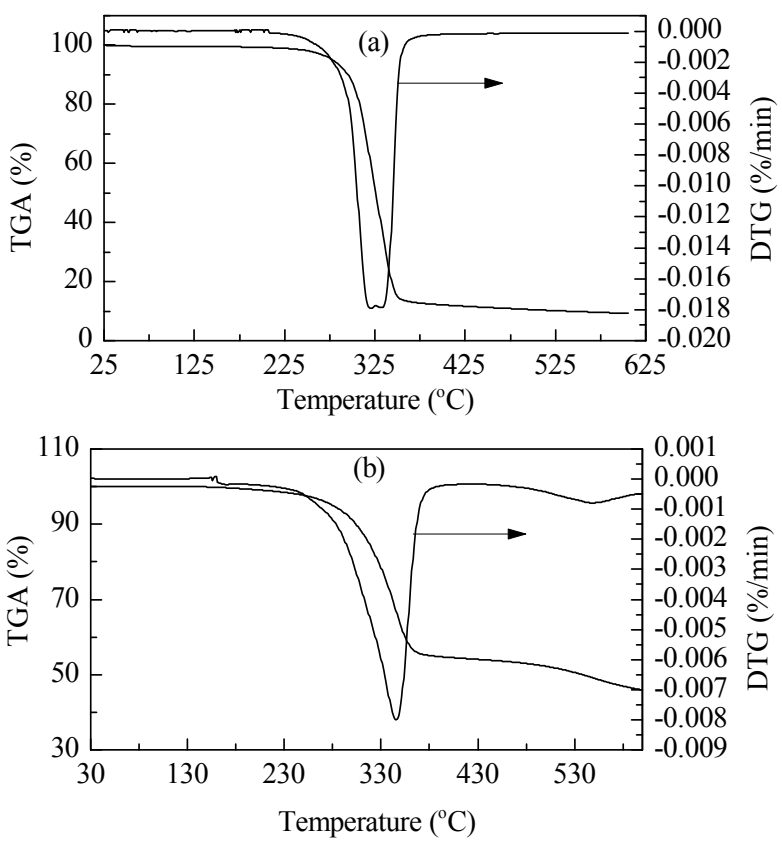

Fig. 1. TGA and DTG curves of [cmmim] $\mathrm{Br}$ (a) and $\left[\mathrm{cmmim}^{\mathrm{c}} \mathrm{BF}_{4}(\mathrm{~b})\right.$.

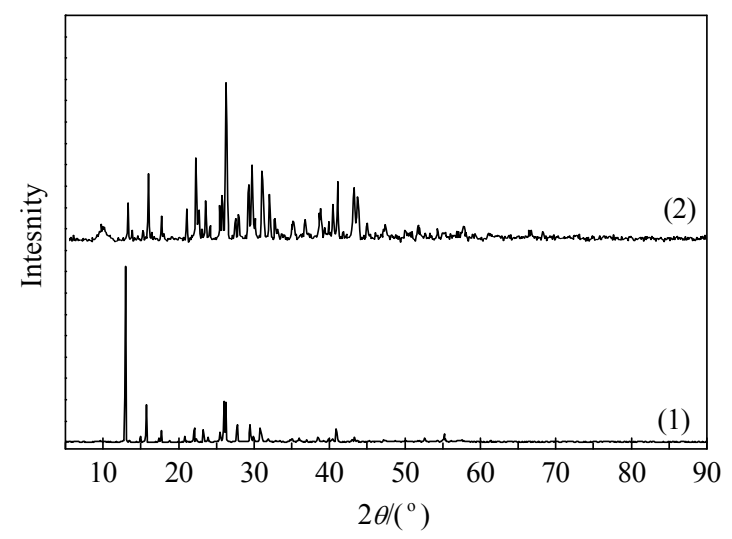

Fig. 2. XRD patterns of [cmmim] $\mathrm{Br}(1)$ and $\left[\mathrm{cmmim}^{\mathrm{B}} \mathrm{BF}_{4}(2)\right.$. 
Table 1

The effect of the amount of the catalysts and temperature on the condensation of $\beta$-naphthol $(2 \mathrm{mmol})$ with 3 -nitrobenzaldehyde $(1 \mathrm{mmol})$.

\begin{tabular}{|c|c|c|c|c|c|c|}
\hline \multirow[b]{2}{*}{ Entry } & \multirow{2}{*}{$\begin{array}{l}\text { Catalyst } \\
\text { amount } \\
(\mathrm{mol} \%)\end{array}$} & \multirow[b]{2}{*}{$\begin{array}{c}T \\
\left({ }^{\circ} \mathrm{C}\right)\end{array}$} & \multicolumn{2}{|c|}{$[\mathrm{cmmim}] \mathrm{Br}$} & \multicolumn{2}{|c|}{$\left[\mathrm{cmmim} \mathrm{BF}_{4}\right.$} \\
\hline & & & $\begin{array}{l}\text { Time } \\
\text { (min) }\end{array}$ & $\begin{array}{c}\text { Yield }^{a} \\
(\%)\end{array}$ & $\begin{array}{l}\text { Time } \\
\text { (min) }\end{array}$ & $\begin{array}{c}\text { Yield }^{2} \\
(\%)\end{array}$ \\
\hline 1 & 10 & 70 & 70 & 32 & 140 & 21 \\
\hline 2 & 10 & 80 & 60 & 54 & 120 & 36 \\
\hline 3 & 10 & 90 & 45 & 74 & 120 & 53 \\
\hline 4 & 10 & 100 & 40 & 83 & 100 & 57 \\
\hline 5 & 10 & 115 & 20 & 93 & 90 & 65 \\
\hline 6 & 10 & 120 & 20 & 93 & 90 & 68 \\
\hline 7 & 7.5 & 115 & 20 & 87 & 90 & 60 \\
\hline 8 & 5 & 115 & 45 & 81 & 110 & 52 \\
\hline 9 & 15 & 115 & 20 & 93 & 80 & 75 \\
\hline
\end{tabular}

a Isolated yield.

$23.2^{\circ}, 25.4^{\circ}, 25.9^{\circ}, 27.6^{\circ}, 29.4^{\circ}, 30.8^{\circ}, 31.7^{\circ}, 32.5^{\circ}, 34.90^{\circ}, 36.5^{\circ}$, $38.6^{\circ}, 40.8^{\circ}, 43.0^{\circ}, 43.5^{\circ}, 44.8^{\circ}$ and several small lines from $45^{\circ}$ to $70^{\circ}$ (Fig. 2).

Next, we studied the efficacy of the catalysts in the synthesis of 14-aryl-14H-dibenzo[a,j]xanthenes. To optimize the reaction conditions, the solvent-free condensation of 2-naphthol with 3-nitrobenzaldehyde was use as a test reaction. Different amounts of [cmmim] Br and [ $\mathrm{cmmim}^{\mathrm{cm}} \mathrm{BF}_{4}$ were used at different temperatures from 70 to $120{ }^{\circ} \mathrm{C}$. The best results were obtained using $10 \mathrm{~mol} \%$ of $[\mathrm{cmmim}] \mathrm{Br}$ at $115^{\circ} \mathrm{C}$. Increasing the reaction time did not further improve the results (Table 1). Moreover, the addition of acetic acid, in comparison with the acetic acid functionalized ionic liquid, was also investigated on the model reaction. Due to the low boiling point of acetic acid $\left(118^{\circ} \mathrm{C}\right)$, acetic acid was used as the catalyst in the model reaction at $115{ }^{\circ} \mathrm{C}$. The expected product was produced in just $50 \%$ yield after $40 \mathrm{~min}$. This shows the superiority of the acetic acid functionalized ionic liquids over acetic acid.

To explore the scope of the catalyst, [cmmim]Br (10 mol\%) was used in the solvent-free with different aromatic aldehydes to prepare a series of 14-aryl-14H-dibenzo[a,j]xanthenes (Table 2). Various aromatic aldehydes containing electron-donating, electron-withdrawing, or halogen substituents on the aromatic ring were utilized successfully in the reaction, and gave the corresponded products in high yields and in short reaction time.

A possible mechanism is also presented here (Scheme 2). First, the aromatic aldehyde is activated by the acidic group of the catalyst to produce $\mathbf{I}$. $\beta$-naphthol then attacks the carbonyl group of the activated aldehyde, which gives the intermediate II. Next, $\mathrm{H}_{2} \mathrm{O}$ leaves giving ortho-quinone methide (o-QM, III). Intermediate III is again activated by [ $\mathrm{cmmim}] \mathrm{Br}$ to give IV which is a Michael acceptor. Michael addition of another $\beta$-naphthol to IV affords $\mathbf{V}$. V converts to VI by tautomerization. Finally, ring condensation occurs to give 14-aryl-14H-dibenzo[a,j]xanthenes.

This mechanism implies that hydrobromic acid could be generated during the reaction. Accordingly, the catalytic application of hydrobromic acid, in comparison with acetic acids functionalized ionic liquid, was studied on model reaction. Due to its low boiling point $\left(122^{\circ} \mathrm{C}\right)$, hydrobromic acid was used as
Table 2

The solvent-free synthesis of 14-aryl-14H-dibenzo[a,j]xanthenes using [cmmim]Br.

\begin{tabular}{lcccc}
\hline Entry & $\mathrm{R}$ & $\begin{array}{c}\text { Time } \\
(\mathrm{min})\end{array}$ & $\begin{array}{c}\text { Yield }^{\mathrm{a}} \\
(\%)\end{array}$ & MP $\left({ }^{\circ} \mathrm{C}\right)$ \\
\hline 1 & $\mathrm{C}_{6} \mathrm{H}_{5}$ & 30 & 90 & $183-184(184-185){ }^{\mathrm{b}}$ \\
2 & $2-\mathrm{ClC}_{6} \mathrm{H}_{4}$ & 25 & 86 & $215-216(209-211)$ \\
3 & $3-\mathrm{ClC}_{6} \mathrm{H}_{4}$ & 25 & 88 & $174-175(207-209)$ \\
4 & $4-\mathrm{ClC}_{6} \mathrm{H}_{4}$ & 20 & 91 & $288-289(284-286)$ \\
5 & $2,3-$ dichloroC $_{6} \mathrm{H}_{3}$ & 20 & 94 & $286-287$ \\
6 & $3-\mathrm{BrC}_{6} \mathrm{H}_{4}$ & 21 & 89 & $189-190(186-188)$ \\
7 & $4-\mathrm{BrC}_{6} \mathrm{H}_{4}$ & 21 & 90 & $296-297$ \\
8 & $4-\mathrm{FC}_{6} \mathrm{H}_{4}$ & 20 & 91 & $238-239$ \\
9 & $3-\mathrm{O}_{2} \mathrm{NC}_{6} \mathrm{H}_{4}$ & 20 & 93 & $210-211(215-217)$ \\
10 & $4-\mathrm{O}_{2} \mathrm{NC}_{6} \mathrm{H}_{4}$ & 17 & 95 & $310-311(312-314)$ \\
11 & $4-\mathrm{MeC}_{6} \mathrm{H}_{4}$ & 22 & 85 & $228-229(225-227)$ \\
12 & $4-\mathrm{HO}_{4}-3-\mathrm{MeOC}_{6} \mathrm{H}_{4}$ & 22 & 83 & $211-212$ \\
13 & $4-\mathrm{PhC}_{6} \mathrm{H}_{4}$ & 30 & 84 & $281-282$ \\
14 & $\mathrm{PhCH}_{2} \mathrm{CH}_{2}$ & 30 & 85 & $176-177$ \\
15 & $4-\mathrm{Cl}_{2}-\mathrm{O}_{2} \mathrm{NC}_{6} \mathrm{H}_{4}$ & 15 & 95 & $245-246$ \\
\hline
\end{tabular}

${ }^{\mathrm{a}}$ Isolated yield. ${ }^{\mathrm{b}}$ Data in the brackets are from Ref. [16].

catalyst in this reaction at $115{ }^{\circ} \mathrm{C}$. The desired product was produced in $58 \%$ of yield after $45 \mathrm{~min}$. This shows that the acetic acid functionalized ionic liquid is responsible for the catalysis in the reaction over any hydrobromic acid present.

Finally, the recyclability of the catalyst was examined in the condensation of 2-naphthol with 3-nitrobenzaldehyde. When the reaction was complete, $\mathrm{H}_{2} \mathrm{O}$ was added to the reaction mixture, stirred and refluxed for $3 \mathrm{~min}$. The reaction mixture was filtered and the water in the filtrate was removed under re-
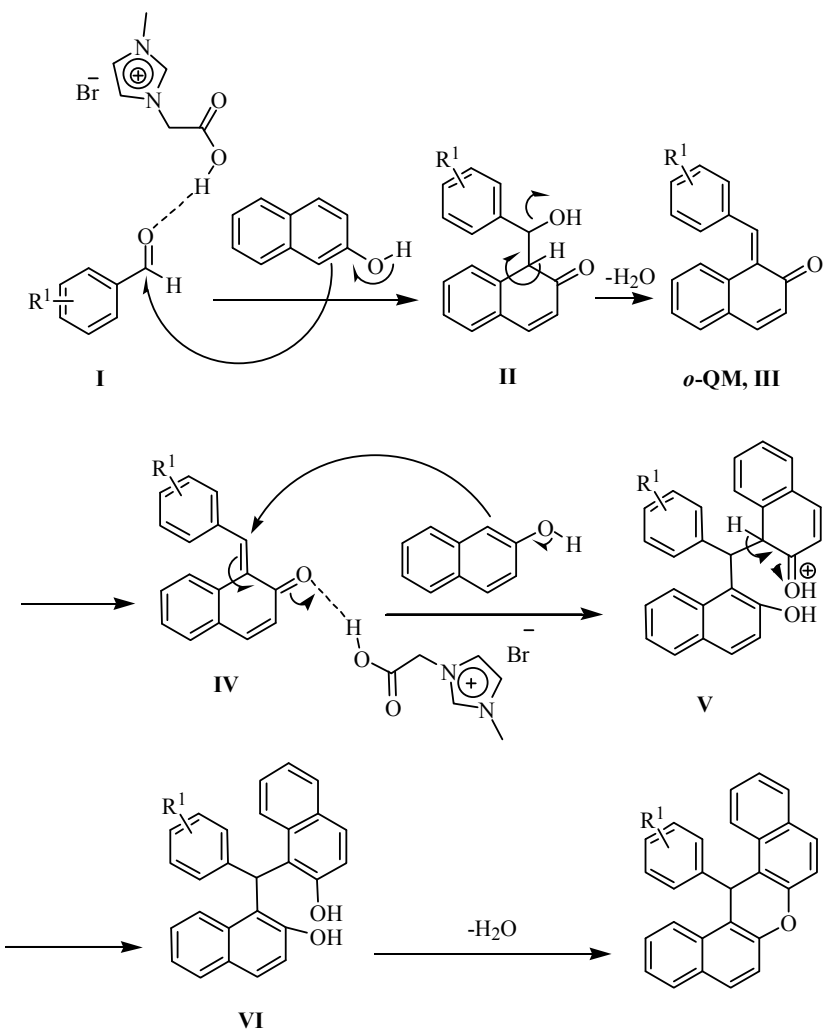

Scheme 2. The proposed mechanism for the synthesis of 14-aryl-14Hdibenzo[a,j]xanthenes using [cmmim]Br. 


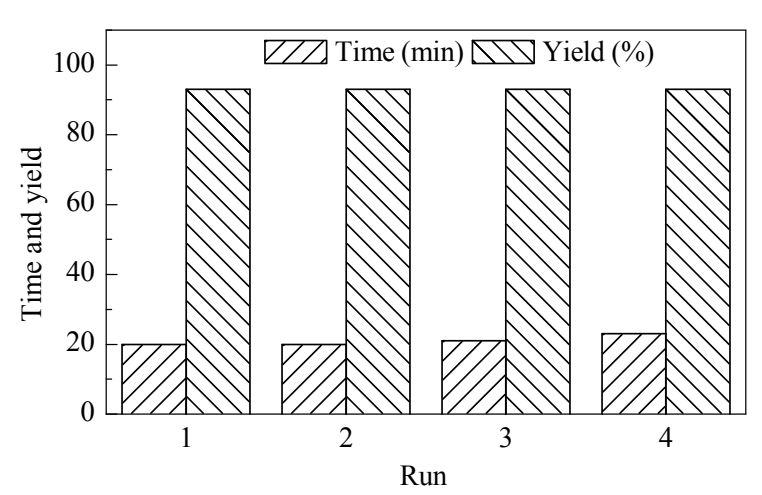

Fig. 3. The condensation of 2-naphthol with dimedone and 3-nitrobenzaldehyde in the presence of reused [cmmim] $\mathrm{Br}(10 \mathrm{~mol} \%)$ in solvent-free conditions at $115^{\circ} \mathrm{C}$.

duced pressure to separate the catalyst from crude product. The reused catalyst was then used in another reaction. The catalytic activity of the catalyst was restored to within the limits of experimental error for four successive runs (Fig. 3).

To compare the applicability and efficiency of $[\mathrm{cmmim}] \mathrm{Br}$ with other reported catalysts in the synthesis of 14 -aryl- $14 \mathrm{H}$ dibenzo[a,j]xanthenes, we have tabulated the TOF of these catalysts in the condensation reaction of $\beta$-naphthol with 3-nitrobenzaldehyde (Table 3). [cmmim] $\mathrm{Br}$ is superior to the previously reported catalysts, in terms of TOF.

\section{Conclusions}

In summary, we have used acetic acid functionalized imidazolium salts as the catalyst for the synthesis of 14 -aryl-14Hdibenzo[a,j]xanthenes from the condensation of 2-naphtol with arylaldehydes. Recovery of the catalyst, easy purification, high yields, short reaction time and higher TOF over the catalyst than the reported catalyst are some important advantages.

\section{References}

[1] Wasserscheid P, Keim W. Angew Chem Int Ed, 2000, 39: 3772

[2] Parvulescu V I, Hardacre C. Chem Rev, 2007, 107: 2615

[3] Earle M J, Katdare S P, Seddon K R. Org Lett, 2004, 6: 707

[4] Ranu B C, Banerjee S. J Org Chem, 2005, 70: 4517

[5] Ranu B C, Adak L, Banerjee S. Can J Chem, 2007, 85: 366

[6] Pegot B, Vo-Thanh G, Gori D, Loupy A. Tetrahedron Lett, 2004, 45: 6425
[7] Zare A, Moosavi-Zare A R, Hasaninejad A, Parhami A, KhalafiNezhad A, Beyzavi M H. Synth Commun, 2009, 39: 3156

[8] Zare A, Parhami A, Moosavi-Zare A R, Hasaninejad A, KhalafiNezhad A, Beyzavi M H, Can J Chem, 2009, 87: 416

[9] Hasaninejad A, Zare A, Shekouhy M, Ameri Rad J. J Comb Chem, 2010, 12: 844

[10] Zolfigol M A, Khazaei A, Moosavi-Zare A R, Zare A. Org Prep Proced Int, 2010, 42: 95

[11] Zolfigol M A, Khazaei A, Moosavi-Zare A R, Zare A.J Iran Chem Soc, 2010, 7: 646

[12] Khazaei A, Zolfigol M A, Moosavi-Zare A R, Zare A. Sci Iran, Trans C, 2010, 17: 31

[13] Zolfigol M A, Khazaei A, Moosavi-Zare A R, Zare A, Kruger H G, Asgari Z, Khakyzadeh V, Kazem-Rostami M. J Org Chem, 2012, 77: 3640

[14] Zolfigol M A, Khazaei A, Moosavi-Zare A R, Zare A, Khakyzadeh V. Appl Catal A, 2011, 400: 70

[15] Khazaei A, Zolfigol M A, Moosavi-Zare A R, Zare A, Ghaemi E, Khakyzadeh V, Asgari Zh, Hasaninejad A. Sci Iran, Trans C, 2011, 18: 1365

[16] Zolfigol M A, Khakyzadeh V, Moosavi-Zare A R, Zare A, Azimi S B, Asgari Z, Hasaninejad A. C R Chim, 2012, 15: 719

[17] Zare A, Yousofi T, Moosavi-Zare A R. RSC Adv, 2012, 2: 7988

[18] Khazaei A, Zolfigol M A, Moosavi-Zare A R, Asgari Z, Shekouhy M, Zare A, Hasaninejad A. RSC Adv, 2012, 2: 8010

[19] Zolfigol M A, Khakyzadeh V, Moosavi-Zare A R, Chehardoli G, Derakhshan-Panah F, Zare A, Khaledian O. Sci Iran Trans C, 2012, 19: 1584

[20] Zare A, Moosavi-Zare A R, Merajoddin M, Zolfigol M A, Hekmat-Zadeh T, Hasaninejad A, Khazaei A, Mokhlesi M, Khakyzadeh V, Derakhshan-Panah F, Beyzavi M H, Rostami E, Arghoon A, Roohandeh R.J Mol Liq, 2012, 167: 69

[21] Zare A, Hekmat-Zadeh T, Mirzaei-Monfared S, Merajoddin M, Torabi-Monfared H, Zolfigol M A, Moosavi-Zare A R, Rostami E, Mokhlesi M, Derakhshan-Panah F, Porbahi S, Balandeh S. S Afr J Chem, 2012, 65: 63

[22] Zare A, Abi F, Moosavi-Zare A R, Beyzavi M H, Zolfigol M A. J Mol Liq, 2013, 178: 113

[23] Moosavi-Zare A R, Zolfigol M A, Zarei M, Zare A, Khakyzadeh V.J Mol Liq, 2013, 186: 63

[24] Khazaei A, Zolfigol M A. Moosavi-Zare A R, Afsar J, Zare A, Khakyzadeh V, Beyzavi M H. Chin J Catal (催化学报), 2013, 34: 1936

[25] Moosavi-Zare A R, Zolfigol M A, Zarei M, Zare A, Khakyzadeh V, Hasaninejad A. Appl Catal A, 2013, 467: 61

[26] Hideo T. JP Patent 56005480. 1981

[27] Lambert R W, Martin J A, Merrett J H, Parkes K E B, Thomas G J. WO Patent 9706178. 1997

[28] Poupelin J P, Saint-Ruf G, Foussard-Blanpin O, Narcisse G, Uchida-

Table 3

Comparison of the results of the condensation of 2-naphthol with 3-nitrobenzaldehyde using [cmmim] Br with those obtained by the recently reported catalysts.

\begin{tabular}{|c|c|c|c|c|c|}
\hline Reaction conditions & Catalyst loading (mol\%) & Time (min) & Yield a $(\%)$ & TOF b $\left(\mathrm{min}^{-1}\right)$ & Ref. \\
\hline [cmmim] Br, solvent-free, $115^{\circ} \mathrm{C}$ & 10 & 20 & 93 & 0.465 & this work \\
\hline $\mathrm{TaCl}_{5}$, reflux conditions, 1 ,2-dichloroethane & 10 & 60 & 93 & 0.155 & [13] \\
\hline $\mathrm{I}_{2}, 90^{\circ} \mathrm{C}$, solvent-free & 10 & 180 & 92 & 0.051 & [16] \\
\hline $\mathrm{HClO}_{4}-\mathrm{SiO}_{2}$, reflux conditions, 1,2-dichloroethane & 1 & 720 & 88 & 0.122 & [17] \\
\hline $\mathrm{CyCl}, 110^{\circ} \mathrm{C}$, solvent-free & 20 & 45 & 90 & 0.100 & [19] \\
\hline $\mathrm{Al}\left(\mathrm{HSO}_{4}\right)_{3}, 125^{\circ} \mathrm{C}$, solvent-free & 16 & 39 & 91 & 0.145 & [25] \\
\hline $\mathrm{P}_{2} \mathrm{O}_{5}, 80^{\circ} \mathrm{C}$, solvent-free & 20 & 52 & 90 & 0.086 & {$[26]$} \\
\hline $\mathrm{InCl}_{3}, 80^{\circ} \mathrm{C}$, solvent-free & 30 & 60 & 82 & 0.045 & [26] \\
\hline
\end{tabular}

${ }^{\mathrm{a}}$ Isolated yield. ${ }^{\mathrm{b}}$ Turnover frequency. 


\section{Graphical Abstract}

Chin. J. Catal., 2014, 35: 573-578 doi: 10.1016/S1872-2067(14)60030-9

Condensation of 2-naphtol with arylaldehydes using acetic acids functionalized ionic liquid as highly efficient and reusable catalysts

Ahmad Reza Moosavi-Zare*, Mohammad Ali Zolfigol*, Omid Khaledian, Vahid Khakyzadeh

University of Sayyed Jamaleddin Asadabadi, Iran

Bu Ali Sina University, Iran

The catalytic activity of acetic acid functionalized imidazolium salts as reusable catalysts were investigated for the synthesis of 14 -aryl-14Hdibenzo[a,j]xanthenes. Thermal gravimetric analysis and powder X-ray diffraction patterns of the catalysts have been studied in this investigation.

Ernouf G, Lacroix R, Europ J Med Chem, 1978, 13: 67

[29] Ion R M, Planner A, Wiktorowicz K, Frackowiak D. Acta Biochim Pol, 1998, 45: 833

[30] Menchen S M, Benson S C, Lam J Y L, Zhen W G, Sun D Q, Rosenblum B B, Khan S H, Taing M. US Patent 6583168. 2003

[31] Bekaert A, Andrieux J, Plat M. Tetrahedron Lett, 1992, 33: 2805

[32] Alcantara-Licudine J P, Kawate M K, Li Q X. J Agric Food Chem, 1997, 45: 766

[33] Pasha M A, Jayashankara V P. Bioorg Med Chem Lett, 2007, 17: 621

[34] Rajitha B, Kumar B S, Reddy Y T, Reddy P N, Sreenivasulu N. Tetrahedron Lett, 2005, 46: 8691

[35] Hong M, Cai C. J Fluorine Chem, 2009, 130: 989

[36] Sarma R J, Baruah J B. Dyes Pigments, 2005, 64: 91

[37] Khosropour A R, Khodaei M M, Moghannian H. Synlett, 2005: 955

[38] Bhattacharya A K, Rana K C, Mujahid M, Sehar I, Saxena A K. Bioorg Med Chem Lett, 2009, 19: 5590
[39] Shakibaei G I, Mirzaei P, Bazgir A. Appl Catal A, 2007, 325: 188

[40] Mahdavinia G H, Rostamizadeh S, Amani A M, Emdadi Z. Ultrason Sonochem, 2009, 16: 7

[41] Das B, Ravikanth B, Ramu R, Laxminarayana K, Rao B V.J Mol Catal A, 2006, 255: 74

[42] Bigdeli M A, Heravi M M, Mahdavinia G H.J Mol Catal A, 2007, 275: 25

[43] Amini M M, Seyyedhamzeh M, Bazgir A. Appl Catal A, 2007, 323: 242

[44] Bigdeli M A, Heravi M M, Mahdavinia G H. Catal Commun, 2007, 8: 1595

[45] Su W K, Yang D, Jin C, Zhang B. Tetrahedron Lett, 2008, 49: 3391

[46] Makaev F, Styngach E, Shargarovskii V, Bets L, Vlad L, Barba A. Russ J Org Chem, 2010, 46: 610

[47] Fei Z F, Zhao D B, Geldbach T J, Scopelliti R, Dyson P J. Chem Eur J, 2004, 10: 4886 